\title{
IQ differences between patients with first episode psychosis in London and Palermo reflect differences in patterns of cannabis use
}

\author{
Laura Ferraro $^{\text {a, b, *, Robin M. Murray }}{ }^{\text {a, b, c }}$, Marta Di Forti ${ }^{\text {a, c, d, e }}$, Diego Quattrone ${ }^{\text {c, d, e }}$, Giada Tripoli ${ }^{\text {a, b }}$, \\ Lucia Sideli $^{\text {a, b }}$, Daniele La Barbera ${ }^{\text {a, }}$, Caterina La Cascia ${ }^{\text {a, } 1}$ \\ ${ }^{a}$ Department of Biomedicine, Neuroscience and advanced Diagnostics (BiND), Psychiatry Section, University of Palermo. Via Gaetano La Loggia, 1, 90129, Palermo, Italy \\ ${ }^{\mathrm{b}}$ Department of Psychosis Studies, Institute of Psychiatry, King's College London, De Crespigny Park, Denmark Hill, London SE5 8AF, UK \\ c South London and Maudsley NHS Mental Health Foundation Trust, UK \\ d Social, Genetic and Developmental Psychiatry Centre, Institute of Psychiatry, Psychology and Neuroscience, King's College London, London SE5 8AF, UK \\ ${ }^{\mathrm{e}}$ National Institute for Health Research (NIHR), Mental Health Biomedical Research Centre, South London and Maudsley NHS Foundation Trust and King's College London, UK
}

\section{A R T I C L E IN F O}

Article history:

Received 14 April 2018

Received in revised form 20 May 2019

Accepted 10 June 2019

Available online $\mathrm{xxx}$

\section{Keywords:}

Neurodevelopment

Cannabis

Risk factors

Cognition

Multicentric study

\section{A B S T R A C T}

Aims

Cognitive impairment is a possible indicator of neurodevelopmental impairment, but not all psychotic patients are cognitively compromised. It has been suggested that heavy cannabis use may precipitate psychosis in those who show no such compromise. This study compares two samples of patients with first-episode psychosis and their respective non-psychotic controls, in London (UK) and Palermo (Italy), and examines whether different patterns of cannabis use are reflected in differences in IQ.

Methods

The two studies used the same inclusion/exclusion criteria and instruments. The sample comprised 249 subjects from London (106 patients and 143 controls) and 247 subjects from Palermo (120 patients and 127 controls). ANCOVA was performed with IQ as the dependent variable and city and frequency of cannabis use as predictors. This was then repeated with the case group only, by adjusting for relevant confounders.

Results

We found a greater amount of cannabis use in the London sample, compared to Palermo and patients from London had higher IQ than patients from Palermo, a difference that was more significant than that reflected between controls (Frrouptity $(1,402)=7.6, p=0.006$ ). Once corrected for symptomatology and treatment, patients from London who had never used cannabis were similar to patients from Palermo regarding IQ. Thus the higher IQ of patients from London was mainly due to the subgroup of cannabis-using patients $\left(\mathrm{F}_{\text {cannabis*ity }}(2,145)=4.6, \mathrm{p}=0.011\right)$.

Conclusions

We can speculate that a greater amount of cannabis-use may have contributed by precipitating psychosis in patients with a higher IQ in London but less so in patients from Palermo.

(C) 2019

\section{Introduction}

People with schizophrenia tend to show subtle cognitive, social and motor impairments in childhood, possible indicators of neurodevelopmental impairment (Howes and Murray, 2014). The average cognitive impairment in schizophrenia can reach two standard deviations below the mean of healthy controls (Heinrichs and Zakzanis, 1998) and patients usually perform more poorly than would be expected based on their premorbid level (Mesholam-Gately et al.,

\footnotetext{
* Corresponding author at: Via Gaetano La Loggia, 1, 90129 Palermo, Italy.

Email addresses: laura.ferraro@unipa.it (L. Ferraro); robin.murray@kcl.ac.uk (R.M. Murray); marta.diforti@kcl.ac.uk (M. Di Forti); diego.1.quattrone@kcl.ac. uk (D. Quattrone); giada.tripoli@kcl.ac.uk (G. Tripoli); lucia.sideli@unipa.it (L. Sideli); daniele.labarbera@unipa.it (D. La Barbera); erika.lacascia@unipa.it (C. La Cascia)

${ }^{1}$ Prof Daniele La Barbera and Caterina La Cascia equally contributed the work. They both are the last names of this paper.
}

2009), which is generally only $0.4-0.5$ standard deviations below the population average (Khandaker et al., 2011; Woodberry et al., 2008). Lower IQ and other cognitive deficits are present at the onset of illness (Harvey and Bowie, 2003) and stay relatively stable over its course (Bora and Murray, 2014), influenced by negative symptoms (Bora et al., 2009) and to a lesser extent by positive symptoms (Ventura et al., 2010).

Cannabis use has been consistently found to be a risk factor for the later onset of psychosis (Marconi et al., 2016; Murray et al., 2016). We and others have reported that IQ and premorbid IQ are higher in cannabis-using psychotic patients than their non-using counterparts, perhaps reflecting less neurodevelopmental impairment in the former (Potvin et al., 2008; Ringen et al., 2010; Yücel et al., 2012; Ferraro et al., 2013). A better premorbid social adjustment in this subgroup could enable these individuals to more easily acquire and sustain a cannabis habit, and thus expose themselves to this non-neurodevelopmental risk factor (Ferraro et al., 2016, 2013). 
At present, there are few available Italian epidemiological studies on psychosis and even fewer that have considered IQ (Bambini et al., 2016; Bechi et al., 2017) or premorbid IQ (Piovan et al., 2016; Tosato et al., 2013). Furthermore, until recently, no studies had been conducted in the southern part of Italy into psychosis or the role of cannabis. However, our Sicilian-Genetic and Psychosis (S-GAP) epidemiological study conducted in Palermo, the Capital of Sicily, found a lower incidence rate of psychosis (Mulè et al., 2017a), compared to that reported from cities in Northern Europe (McGrath et al., 2004), but similar to Northern Italy (Lasalvia et al., 2014; Tarricone et al., 2012).

We, therefore, decided to examine the relationship between cannabis use and IQ in the S-GAP sample of psychotic patients and population controls, and compare the findings with those from our previous GAP study in London (Ferraro et al., 2013). We were particularly interested to see how any differences in patterns of cannabis use in our two samples might be reflected in cognition.

The aims of the present study were, therefore: 1) to describe for the first time the entire patient/control epidemiologically-derived Palermo sample in terms of IQ and other relevant variables; and 2) to compare patients from the S-GAP study together with their respective controls with patients and controls from the London GAP study, in order to test the effect of different patterns of cannabis use on IQ in the two cities and the interaction terms between these variables.

Precisely, we expected to find (i) a greater amount of cannabis use in the London sample, compared to Palermo; (ii) that the difference in IQ between patients from the two cities is more significant than that reflected between controls; and that (iii) cannabis use in patients from London is associated with a higher IQ, compared to Palermo, once corrected for relevant confounders.

\section{Methods}

\subsection{Subjects}

The sample comprised 496 subjects (226 first-episode psychotic patients; 270 non-psychotic controls). 249 were recruited in London (UK) (106 patients; 143 controls), between February 2006-June 2011 as part of the GAP study (Di Forti et al., 2009; Ferraro et al., 2013); and 247 (120 patients; 127 controls) were recruited in Palermo (Italy) as part of the S-GAP study (Mulè et al., 2017b), between January 2007-September 2014.

Both studies were approved by the local research ethics committees, following the ethical standards of the Declaration of Helsinki. All subjects were asked to sign a consent form, after a full explanation of the procedures of the study and they were informed about the possibility to leave the study at any moment. Data were fully anonymised. The two studies were designed to also explicitly study cannabis use as a risk factor for psychosis (Di Forti et al., 2015, 2014; Mulè et al., $2017 b$ ). Therefore, to avoid any bias, enrolment materials did not mention cannabis or any other drug use.

These two studies used the same inclusion/exclusion criteria and instruments. Subjects were aged 18-65 years and resident in the catchment area for at least six months. Patients met ICD-10 criteria for psychosis (F10-19, F20-F29 and F30-F33) (World Health Organization, 1992a); exclusion criteria were organic psychosis, acute intoxication (F1x.0), learning disabilities, history of traumatic brain injury and lack of speech fluency.

Population controls, representative of the local general population, were recruited through local newspapers and internet advertising, job centres, hospitals and a pre-existing volunteer database.

\subsection{Assessment}

A modified version of the Medical Research Council (MRC) Sociodemographic Schedule (Di Forti et al., 2009) was administered to all subjects. Diagnoses for patients were ascertained using the Schedules for Clinical Assessment in Neuropsychiatry (SCAN, version 2.1) (World Health Organization, 1992b). Psychotic symptoms were assessed by administering the Positive and Negative Syndrome Scale (PANSS) (Kay et al., 1989). IQ was estimated based on five subtests (Information, Digit Span, Matrix, Block Design and Digit Symbol) of the Wechsler Adult Intelligence Scale-Third Edition (WAIS-III) (Velthorst et al., 2013; Wechsler, 1997).

Cannabis use was assessed using the Cannabis Experience Questionnaire (modified version) (Di Forti et al., 2009); skunk, super skunk and sinsemilla were defined "high potency cannabis" (Potter et al., 2008), by using the data published in the EMCDDA (European Monitoring Centre for Drugs and Drug Addiction and Europol, 2016; Trends and developments 2013: Legal notice, 2013). The frequency of use was defined as the amount of cannabis intake in the period of life in which cannabis was mostly used (Di Forti et al., 2009). The Psychosis Screening Questionnaire (PSQ) (Bebbington and Nayani, 1995) was administered to controls to exclude any psychotic symptomatology. Premorbid IQ in Palermo was estimated using the TIB-Test di Intelligenza Breve (Sartori et al., 1997), the Italian version of the NART (Nelson and Willison, 1991).

\subsection{Statistical analysis}

Statistical analyses were carried out using SPSS 25.0. Chi-square $(\chi 2)$ tests and $t$-tests were used for comparisons and (i) to test differences in amount of cannabis-use in the London sample, compared to Palermo. To test (ii) if the difference in IQ between patients from the two cities is more significant than that reflected between controls, we included patients and controls in a first multifactorial ANCOVA corrected by Bonferroni, with IQ as the dependent variable. IQ distribution, overall, respected the assumption of normality; thus parametric tests were used. A priori confounders were confirmed by significance tests (Pearson correlations, $t$-tests and chi-squared tests) to select which of these to include in each analysis, to do not overfit the model. Interaction between city (Palermo, London), group (patients, controls), and frequency of cannabis use (never, less than everyday, everyday) were inserted in the model and sex, ethnicity (self-ascribed and grouped as white, black, other), level of education achieved (no education, Italian secondary first degree education/UK GCSE, college or job related education, degree), living status (alone, with parents, with their own family, other), and employment - a proxy for personal socio-economic-status - (employed, unemployed, student) were used to correct the analysis.

To test (iii) if cannabis use in patients from London is reflected in a higher IQ, compared to Palermo, the ANCOVA was repeated with the patients' group only and PANSS scores and days of medication with antipsychotics at the time of WAIS were additionally included as covariates to avoid any influence of current symptomatology and pharmacotherapy on IQ. Age was not included in either analysis because WAIS scores already take it into account.

We finally performed an exploratory analysis, by repeating in the Palermo sample the analysis previously done on the GAP sample (Ferraro et al., 2013) with premorbid IQ as dependent variable and group and cannabis (yes/no) as independent factors, corrected by age and sex. Bootstrap 95\% confidence intervals were calculated, using 1000 samples, bias-corrected and accelerated. 


\section{Results}

\subsection{Socio-demographic characteristics}

Patients were more likely to be male $\left(\chi^{2}(1)=15.3, \mathrm{p}<0.001\right)$, less educated $\left(\chi^{2}(3)=59.6, \mathrm{p}<0.001\right)$, unemployed $\left(\chi^{2}(2)=47.6, \mathrm{p}<0.001\right)$ and single $\left(\chi^{2}(2)=32.6, \mathrm{p}<0.001\right)$ than controls, in both cities. There were no differences in age between patients and controls, either in Palermo or London.

Participants were comparable between the two cities regarding age, sex, employment status and relationship status overall, but controls from Palermo were more likely to be in a stable relationship, compared to the same group from London $\left(\chi^{2}(2)=8.2, p=0.016\right)$.

Both patients and controls from London were more likely to have higher levels of education $\left(\chi^{2}(3)=35.0, p<0.001\right)$ and to live alone, compared to Palermo, where subjects mostly stayed with their parents $\left(\chi^{2}(3)=50.7, p<0.001\right)$. Patients from London were more likely to belong to minority ethnic groups $\left(\chi^{2}(2)=17.6, \mathrm{p}<0.001\right)$ than controls. In Palermo, $95.8 \%$ of patients and $94.5 \%$ of controls were white Caucasian, Italian born people $\left(\chi^{2}(2)=0.2, \mathrm{p}=0.882\right)$, and thus there was a significant difference between the two cities $\left(\chi^{2}(2)=137.2, p<0.001\right)$ (Table 1).

\subsection{Clinical characteristics and $I Q$}

The Palermo sample comprised a higher percentage of non-affective psychosis $(82.2 \%)$ than the London sample $(72.3 \%)\left(\chi^{2}(1)=4.1\right.$, $\mathrm{p}<0.044$ ) and they were also more symptomatic at the time of the first assessment, with higher scores of positive $(\mathrm{t}(214)=9.6, \mathrm{p}<0.001)$, negative $(\mathrm{t}(214)=6.7, \mathrm{p}<0.001)$ and general $(\mathrm{t}(214)=13.4, \mathrm{p}<0.001)$ symptoms. Patients showed lower IQ than controls, both in Palermo $(\mathrm{t}(245)=-11.4, \mathrm{p}<0.001)$ and in London $(\mathrm{t}(247)=-8.9, \mathrm{p}<0.001)$. Both patients and controls from London had higher IQ compared to the equivalent subjects from Palermo $(\mathrm{t}(471)=-5.9, \mathrm{p}<0.001)$ (Table 2).

\subsection{Different patterns of cannabis use}

\subsubsection{London versus Palermo}

Patients from London were more likely to have used cannabis in their lifetime $\left(\chi^{2}(1)=10.4, p=0.001\right)$, and daily $\left(\chi^{2}(2)=8.2, p=0.017\right)$ than patients from Palermo. Both patients and controls from London were more likely to have used high potency cannabis $\left(\chi^{2}(1)=52.1\right.$, $p<0.001)$ and other illegal drugs in their lifetime $\left(\chi^{2}(1)=24.5\right.$, $\mathrm{p}<0.001)$, than their counterparts from Palermo. Controls from London were more likely to be current smokers - i.e. having smoked cannabis in the previous four weeks $\left(\chi^{2}(1)=7.7, p=0.005\right)$ and they had started smoking cannabis earlier in their life $(\mathrm{t}(302)=2.7$, $\mathrm{p}=0.007)$, compared to Palermo controls.

\subsubsection{Patients versus controls}

There were no statistical differences between patients and controls in lifetime cannabis use in either city (all $\mathrm{p}>0.05$ ). However, patients were more likely than controls to have smoked cannabis daily in both cities (all $\mathrm{p}<0.001)$. Patients from Palermo started smoking cannabis two years earlier than their respective controls $(\mathrm{t}(141)=-0.23$, $\mathrm{p}=0.021)$ and they were more likely to smoke alone (vs social) $\left(\chi^{2}(2)=21.6, \mathrm{p}<0.001\right)$ and to have used other illegal drugs $\left(\chi^{2}(1)=7.1, \mathrm{p}=0.008\right)$ (Table 3$)$.

\subsection{IQ prediction model for patients and controls by city}

The first model included all predictors and revealed a significant relationship between city and group $\left(\mathrm{F}^{\text {group*ity }}(1,402)=7.6, \mathrm{p}=0.006\right)$, meaning that the difference in IQ between patients from Palermo, compared to London (Mean difference $\left(\mathrm{M}^{\mathrm{diff}}\right)=-14.4 ; 95 \%$ C.I. $=-15.4,-13.5)$ was greater than that detected between controls from the two cities $\left(\mathrm{M}^{\mathrm{diff}}=-4.2 ; 95 \%\right.$ C.I. $\left.=-5.2,-3.3\right)$. Sex $(\mathrm{F}(1$, $402)=0.1, p=0.673)$ and employment status $(\mathrm{F}(2,402)=2.4$, $\mathrm{p}=0.089)$ were not related to IQ. Education $(\mathrm{F}(3,402)=8.2$, $\mathrm{p}<0.001)$, ethnicity $(\mathrm{F}(2,402)=6.5, \mathrm{p}=0.002)$ and living status $(\mathrm{F}(3$, $402)=7.5, p<0.001$ ) were, in turn, significant predictors of IQ. The interaction of city and group with frequency of cannabis showed borderline significance $\left(\mathrm{F}^{\text {group*ity*canbis }}(2,402)=3.0, \mathrm{p}=0.05\right)$, which increased once we removed non-significant predictors from the model $\left(\mathrm{F}^{\text {group*city*cannabis }}(2,406)=3.6, \mathrm{p}=0.026\right)$, thus suggesting a role for cannabis use in influencing the relationship between caseness and cities (Fig. 1).

\subsection{What predicts $I Q$ in patients?}

In a preliminary analysis, days of treatment with antipsychotics were associated with positive $(\mathrm{r}=-0.29, \mathrm{p}<0.001)$ and general $(\mathrm{r}=-0.27, \mathrm{p}<0.001)$ symptoms, but not with negative symptoms $(\mathrm{r}=-0.07, \mathrm{p}=0.346)$. Thus, we inserted symptoms and treatment with antipsychotics in two subsequent analyses. Once corrected for education, living status and symptoms, patients from London who smoked cannabis occasionally had higher IQ than non-using patients $\left(M^{\text {diff }}=11.8 ; 95 \%\right.$ C.I. $\left.=1,22.7\right)$, while patients in this last group were similar to patients from Palermo, irrespective to their cannabis use $\left(\mathrm{F}^{\text {cannabis*ity }}(2,182)=3.5, \mathrm{p}=0.030\right)$. Higher education $(\mathrm{F}(3,182)=3.6$, $\mathrm{p}=0.013)$, living alone $(\mathrm{F}(3,182)=3.4, \mathrm{p}=0.018)$, and fewer negative symptoms $(\mathrm{F}(1,182)=5.1, \mathrm{p}=0.024)$ were significantly related to higher IQ, while positive $(\mathrm{F}(1,182) \mathrm{p}=0.349)$, and general symptoms $(\mathrm{F}(2,182)=0.025, \mathrm{p}=0.875)$ were not. When further corrected for days of treatment with antipsychotics, the interaction term between frequency of cannabis use and city stayed significant $\left(\mathrm{F}^{\text {cannabis*ity }}(2,145)=46, \mathrm{p}=0.011\right)$. The only two relevant confounders, related to higher IQ, were higher education $(F(2,145)=2,9, \mathrm{p}=0.037)$ and fewer negative symptoms $(\mathrm{F}(1,145)=4.7, \mathrm{p}=0.031)$ (Fig. 2).

\subsection{Premorbid IQ in Palermo sample}

We also had data for premorbid IQ, collected in 25 patients $($ Mean= 98.1, $\mathrm{sd}=13)$ and 62 controls $($ Mean=102.5, $\mathrm{sd}=18.2)$ from Palermo. Patients had almost four points-lower premorbid IQ than controls, but this difference was not significant $\left(\mathrm{M}^{\mathrm{diff}}=3.7,95 \%\right.$ C.I. $=-0.8,8.2$ ). Both patients and controls who had smoked cannabis in their lifetime had more than eight points higher premorbid IQ than those who did not $\left(\mathrm{M}^{\mathrm{diff}}=8.4,95 \%\right.$ C.I. $\left.=2.7,14\right)$ and no interaction was found between these two terms $\left(\mathrm{F}^{\text {group*annabis }}(1,81)=0.8, \mathrm{p}=0.367\right)$.

\section{Discussion}

\subsection{Comparisons between London and Palermo sample}

Differences in sex, employment, education and relationship status between patients and controls have been associated with psychosis risk; therefore our findings concerning these variables were expected 
Table 1

Socio-demographic characteristics by patient/control status and by city, overall and stratified.

\begin{tabular}{|c|c|c|c|c|c|c|c|c|c|c|c|c|c|c|}
\hline Variables & London & & & & & & Palermo & & & & & & $\begin{array}{l}\text { Patients vs. controls } \\
\text { Total }\end{array}$ & $\begin{array}{l}\text { London Vs. } \\
\text { Palermo }\end{array}$ \\
\hline & Patients & $\mathrm{N}$ & Controls & $\mathrm{N}$ & $\chi^{2}$ or $t$-test (df) & $\mathrm{p}$-Value & Patients & $\mathrm{N}$ & Controls & $\mathrm{N}$ & $\chi^{2}$ or t-test $(\mathrm{df})$ & p-Value & p-Value & $\mathrm{p}$-Value \\
\hline Sex & & 106 & & 143 & $12.6(1)$ & $<0.001$ & & 120 & & 127 & $4.1(1)$ & 0.041 & $<0.001$ & Total $=0.659$ \\
\hline Male, N (\%) & $75(70.8)$ & & $69(48.3)$ & & & & $75(62.5)$ & & $63(49.6)$ & & & & & Patients $=0.190$ \\
\hline Female, N (\%) & $31(29.2)$ & & $74(51.7)$ & & & & $45(37.5)$ & & $64(50.4)$ & & & & & Controls $=0.824$ \\
\hline Age, Mean (sd) & $29.6(8.6)$ & 106 & $29.9(11.1)$ & 143 & $6.9(247)$ & 0.755 & $28.6(10.2)$ & 120 & $31.2(12.3)$ & 127 & $6.4(245)$ & 0.066 & 0.113 & $\begin{array}{l}\text { Total }=0.827 \\
\text { Patients }=0.463 \\
\text { Controls }=0.351\end{array}$ \\
\hline Education & & 106 & & 142 & $17.6(3)$ & 0.001 & & 119 & & 126 & $46.2(3)$ & $<0.001$ & $<0.001$ & Total $<\mathbf{0 . 0 0 1}$ \\
\hline Degree, N (\%) & $21(19.8)$ & & $57(40.1)$ & & & & $4(3.4)$ & & $13(10.3)$ & & & & & Patients $<\mathbf{0 . 0 0 1}$ \\
\hline Vocational/College, N (\%) & $51(48.1)$ & & $65(45.8)$ & & & & $50(42.0)$ & & $94(74.6)$ & & & & & Controls $<\mathbf{0 . 0 0 1}$ \\
\hline 1st degree/CGSE, N (\%) & $22(20.8)$ & & $15(10.6)$ & & & & $47(39.5)$ & & $18(14.3)$ & & & & & \\
\hline No education, $\mathrm{N}(\%)$ & $12(11.3)$ & & $5(3.5)$ & & & & $18(15.1)$ & & $1(0.8)$ & & & & & \\
\hline Ethnicity & & 98 & & 143 & $17.6(2)$ & $<0.001$ & & 120 & & 127 & $0.2(2)$ & 0.882 & 0.082 & Total $<\mathbf{0 . 0 0 1}$ \\
\hline White, N (\%) & $31(31.6)$ & & $83(58.0)$ & & & & $115(95.8)$ & & $120(94.5)$ & & & & & Patients $<\mathbf{0 . 0 0 1}$ \\
\hline Black, N (\%) & $44(44.9)$ & & $45(31.5)$ & & & & $3(2.5)$ & & $4(3.1)$ & & & & & Controls $<\mathbf{0 . 0 0 1}$ \\
\hline Other, N (\%) & $23(23.5)$ & & $15(10.5)$ & & & & $2(1.7)$ & & $3(2.4)$ & & & & & \\
\hline Employment & & 93 & & 108 & $27.6(2)$ & $<0.001$ & & 120 & & 127 & $21.8(2)$ & $<0.001$ & $<0.001$ & Total $=0.186$ \\
\hline No, Unemployed, N (\%) & $54(58.1)$ & & $26(24.1)$ & & & & $66(55.0)$ & & $33(26.0)$ & & & & & Patients $=0.638$ \\
\hline No, Student, N (\%) & $6(6.5)$ & & $27(25.0)$ & ( & & & $19(15.8)$ & & $37(29.1)$ & & & & & Controls $=0.097$ \\
\hline Employed, N (\%) & $33(43.8)$ & & $55(50.9)$ & & & & $35(29.2)$ & & $57(44.9)$ & & & & & \\
\hline Relationship & & 93 & & 107 & $9.03(2)$ & 0.011 & & 119 & & 127 & $26.5(2)$ & $<0.001$ & $<0.001$ & Total $=0.055$ \\
\hline Single, N (\%) & $68(73.1)$ & & $60(56.1)$ & & & & $83(69.7)$ & & $49(38.6)$ & & & & & Patients $=0.763$ \\
\hline In a relationship, $\mathrm{N}(\%)$ & $22(23.7)$ & & $46(43.0)$ & & & & $30(25.2)$ & & $73(57.5)$ & & & & & Controls $=\mathbf{0 . 0 1 6}$ \\
\hline Divorced/widowed, N (\%) & $3(3.2)$ & & $1(0.9)$ & & & & $6(5.0)$ & & $5(3.9)$ & & & & & \\
\hline Living status & & 94 & & 108 & $20.0(3)$ & $<0.001$ & & 119 & & 126 & $18.7(3)$ & $<0.001$ & $<0.001$ & Total $<\mathbf{0 . 0 0 1}$ \\
\hline Alone, N (\%) & $35(37.2)$ & & $15(13.9)$ & & & & $3(2.5)$ & & $11(8.7)$ & & & & & Patients $<\mathbf{0 . 0 0 1}$ \\
\hline My own family/part of, $\mathrm{N}(\%)$ & $15(16.0)$ & & $33(30.6)$ & & & & $23(19.3)$ & & $40(31.7)$ & & & & & Controls $=\mathbf{0 . 0 3 5}$ \\
\hline Parents, N (\%) & $29(30.9)$ & & $28(25.9)$ & & & & $81(68.1)$ & & $52(41.3)$ & & & & & \\
\hline Other family/other, $\mathrm{N}(\%)$ & $15(16.0)$ & & $32(29.6)$ & & & & $12(10.1)$ & & $23(18.3)$ & & & & & \\
\hline
\end{tabular}

Abbreviations: $\mathrm{df}=$ degree of freedom; $\mathrm{N}=$ number; $\mathrm{sd}=$ standard deviation 
Table 2

Clinical characteristics of patients by city, and IQ by patient/control status and by city.

\begin{tabular}{|c|c|c|c|c|c|c|c|c|c|c|}
\hline & London & $\mathrm{N}$ & $t$-test (df) & $\begin{array}{l}\text { p- } \\
\text { Value }\end{array}$ & Palermo & $\mathrm{N}$ & $t$-test (df) & $\begin{array}{l}\mathrm{p}- \\
\text { Value }\end{array}$ & $\begin{array}{l}\text { Patients vs. controls } \\
\text { p-Value }\end{array}$ & $\begin{array}{l}\text { London vs. Palermo } \\
\text { p-Value }\end{array}$ \\
\hline Diagnosis & & 119 & & & & 169 & & & & $<0.044$ \\
\hline Non affective, $\mathrm{N}(\%)$ & $86(72.3)$ & & - & - & $139(82.2)$ & & - & - & - & \\
\hline Affective, N (\%) & $33(27.7)$ & & - & 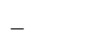 & $30(17.8)$ & & - & - & - & \\
\hline PANSS & & 100 & & & & 116 & & & & \\
\hline Positive, mean (sd) & $14.6(6.1)$ & & - & - & $23.3(6.8)$ & & - & - & - & $<0.001$ \\
\hline Negative, mean (sd) & $14.2(5.2)$ & & - & - & $21.9(10.3)$ & & - & - & - & $<0.001$ \\
\hline General, mean (sd) & $29.3(6.8)$ & & - & - & $50.3(14.2)$ & & - & - & 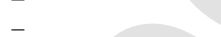 & $<0.001$ \\
\hline IQ & & & & $<0.001$ & & & & $<0.001$ & $<0.001$ & $<0.001$ \\
\hline Patients, mean (sd) & $87.9(16.1)$ & 106 & $-8.9(247)$ & & $73.4(18.2)$ & 120 & $-11.4(245)$ & & & Patients $<\mathbf{0 . 0 0 1}$ \\
\hline Controls, mean (sd) & $106.6(16.2)$ & 143 & & & $100.7(19.0)$ & 127 & & & & Controls $=\mathbf{0 . 0 0 6}$ \\
\hline
\end{tabular}

Abbreviations: $\mathrm{IQ}=$ intellectual quotient; $\mathrm{df}=$ degree of freedom; $\mathrm{N}=$ number; $\mathrm{sd}=$ standard deviation .

Table 3

Pattern of cannabis use by patient/control status and by city.

\begin{tabular}{|c|c|c|c|c|c|c|c|c|c|c|c|c|c|c|}
\hline Variables & London & & & & & & Palermo & & & & & & $\begin{array}{l}\text { Patients } \\
\text { vs. } \\
\text { controls } \\
\text { Total }\end{array}$ & $\begin{array}{l}\text { London vs. } \\
\text { Palermo }\end{array}$ \\
\hline & Patients & $\mathrm{N}$ & Controls & $\mathrm{N}$ & $\begin{array}{l}\chi^{2} \text { or } \\
\text { t-test } \\
(\mathrm{df})\end{array}$ & $\begin{array}{l}\mathrm{p}- \\
\text { Value }\end{array}$ & Patients & $\mathrm{N}$ & Controls & $\mathrm{N}$ & $\begin{array}{l}\chi^{2} \text { or } \\
\text { t-test } \\
\text { (df) }\end{array}$ & $\begin{array}{l}\mathrm{p}- \\
\text { Value }\end{array}$ & p-Value & p-Value \\
\hline $\begin{array}{l}\text { Cannabis use } \\
\text { lifetime }\end{array}$ & & 106 & & 143 & $\begin{array}{l}3.7 \\
(1)\end{array}$ & 0.052 & & 120 & & 127 & $\begin{array}{l}3.7 \\
(1)\end{array}$ & 0.054 & 0.870 & Total $=0.068$ \\
\hline Yes, N (\%) & $\begin{array}{l}77 \\
(72.6)\end{array}$ & & $\begin{array}{l}87 \\
(60.8)\end{array}$ & & & & $\begin{array}{l}62 \\
(51.7)\end{array}$ & & $\begin{array}{l}81 \\
(63.8)\end{array}$ & & & & & Patients $=0.001$ \\
\hline No, N (\%) & $\begin{array}{l}29 \\
(27.4)\end{array}$ & & $\begin{array}{l}56 \\
(39.2)\end{array}$ & & & & $\begin{array}{l}58 \\
(48.3)\end{array}$ & & $\begin{array}{l}46 \\
(36.2)\end{array}$ & & & & & Controls $=0.619$ \\
\hline $\begin{array}{l}\text { Age at first use, } \\
\text { Mean (sd) }\end{array}$ & $\begin{array}{l}16.3 \\
(4.3)\end{array}$ & 73 & $\begin{array}{l}15.8 \\
(3.1)\end{array}$ & 87 & $\begin{array}{l}0.7 \\
(158)\end{array}$ & 0.428 & $\begin{array}{l}16.5 \\
(4.7)\end{array}$ & 62 & $\begin{array}{l}18.4 \\
(4.5)\end{array}$ & 81 & $\begin{array}{l}-0.23 \\
(141)\end{array}$ & 0.021 & 0.095 & $\begin{array}{l}\text { Total }=\mathbf{0 . 0 0 2} \\
\text { Patients }=0.752 \\
\text { Controls }<\mathbf{0 . 0 0 1}\end{array}$ \\
\hline $\begin{array}{l}\text { Frequency of } \\
\quad \text { use }\end{array}$ & & 98 & & 128 & $\begin{array}{l}16.5 \\
(2)\end{array}$ & $<0.001$ & & 119 & & 124 & $\begin{array}{l}25.6 \\
(2)\end{array}$ & $<0.001$ & $<0.001$ & Total $=0.242$ \\
\hline Never, N (\%) & $\begin{array}{l}29 \\
(29.6)\end{array}$ & & $\begin{array}{l}56 \\
(43.8)\end{array}$ & & & & $\begin{array}{l}58 \\
(48.7)\end{array}$ & & $\begin{array}{l}46 \\
(37.1)\end{array}$ & & & & & Patients $=\mathbf{0 . 0 1 7}$ \\
\hline $\begin{array}{l}\text { Less than } \\
\text { everyday, } \mathrm{N} \\
(\%)\end{array}$ & $\begin{array}{l}37 \\
(37.8)\end{array}$ & & $\begin{array}{l}58 \\
(45.3)\end{array}$ & & & & $\begin{array}{l}33 \\
(27.7)\end{array}$ & & $\begin{array}{l}70 \\
(56.5)\end{array}$ & & & & & Controls $=0.159$ \\
\hline $\begin{array}{l}\text { Everyday, N } \\
(\%)\end{array}$ & $\begin{array}{l}32 \\
(32.7)\end{array}$ & & $\begin{array}{l}14 \\
(10.9)\end{array}$ & & & & $\begin{array}{l}28 \\
(23.5)\end{array}$ & & $8(6.5)$ & & & & & \\
\hline Current use & & 78 & & 88 & $\begin{array}{l}0.7 \\
(1)\end{array}$ & 0780 & & 112 & & 114 & $\begin{array}{l}1.3 \\
(1)\end{array}$ & 0.246 & 0.343 & Total $=\mathbf{0 . 0 0 5}$ \\
\hline Yes, N (\%) & $\begin{array}{l}30 \\
(38.5)\end{array}$ & & $\begin{array}{l}32 \\
(36.4)\end{array}$ & & & & $\begin{array}{l}31 \\
(27.7)\end{array}$ & & $\begin{array}{l}24 \\
(21.1)\end{array}$ & & & & & Patients $=0.117$ \\
\hline No, N (\%) & $\begin{array}{l}48 \\
(61.5)\end{array}$ & & $\begin{array}{l}56 \\
(63.6)\end{array}$ & & & & $\begin{array}{l}81 \\
(72.3)\end{array}$ & & $\begin{array}{l}90 \\
(78.9)\end{array}$ & & & & & Controls $=\mathbf{0 . 0 1 6}$ \\
\hline $\begin{array}{l}\text { Type of } \\
\text { cannabis used }\end{array}$ & & 54 & & 71 & $\begin{array}{l}1.4 \\
(1)\end{array}$ & 0.226 & & 58 & & 69 & 0.8 & 0.367 & 0.736 & Total $<\mathbf{0 . 0 0 1}$ \\
\hline $\begin{array}{l}\text { Hash/herbal, } \\
\text { N (\%) }\end{array}$ & $\begin{array}{l}23 \\
(42.6)\end{array}$ & & $\begin{array}{l}38 \\
(53.5)\end{array}$ & & & & $\begin{array}{l}54 \\
(93.1)\end{array}$ & & $\begin{array}{l}61 \\
(88.4)\end{array}$ & & & & & Patients $<\mathbf{0 . 0 0 1}$ \\
\hline $\begin{array}{l}\text { Skunk/super } \\
\text { skunk, N (\%) }\end{array}$ & $\begin{array}{l}31 \\
(57.4)\end{array}$ & & $\begin{array}{l}33 \\
(46.5)\end{array}$ & & & & $4(6.9)$ & & $8(11.6)$ & & & & & Controls $<\mathbf{0 . 0 0 1}$ \\
\hline Mode of use & & 68 & & 82 & $\begin{array}{l}1.7 \\
(2)\end{array}$ & 0.420 & & 60 & & 75 & $\begin{array}{l}21.6 \\
(2)\end{array}$ & $<0.001$ & $<0.001$ & Total $=0.155$ \\
\hline Social, N (\%) & $\begin{array}{l}43 \\
(63.2)\end{array}$ & & $\begin{array}{l}60 \\
(73.2)\end{array}$ & & & & $\begin{array}{l}36 \\
(60.0)\end{array}$ & & $\begin{array}{l}69 \\
(92.0)\end{array}$ & & & & & Patients $=\mathbf{0 . 0 0 7}$ \\
\hline $\begin{array}{l}\text { Isolated, } \mathrm{N} \\
(\%)\end{array}$ & $\begin{array}{l}12 \\
(17.6)\end{array}$ & & $\begin{array}{l}10 \\
(12.2)\end{array}$ & & & & $7(11.7)$ & & $4(5.3)$ & & & & & Controls $=0.372$ \\
\hline Both, N (\%) & $\begin{array}{l}13 \\
(19.1)\end{array}$ & & $\begin{array}{l}12 \\
(14.6)\end{array}$ & 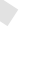 & & & $\begin{array}{l}17 \\
(28.3)\end{array}$ & & $2(2.7)$ & & & & & \\
\hline Other drugs & & 101 & & 137 & $\begin{array}{l}1.4 \\
(1)\end{array}$ & 0.223 & & 118 & & 124 & $\begin{array}{l}7.1 \\
(1)\end{array}$ & 0.008 & 0.026 & Total $<\mathbf{0 . 0 0 1}$ \\
\hline Yes, N (\%) & $\begin{array}{l}44 \\
(43.6)\end{array}$ & & $\begin{array}{l}49 \\
(35.8)\end{array}$ & & & & $\begin{array}{l}30 \\
(25.4)\end{array}$ & & $\begin{array}{l}15 \\
(12.1)\end{array}$ & & & & & Patients $=\mathbf{0 . 0 0 5}$ \\
\hline No, N (\%) & $\begin{array}{l}57 \\
(56.4)\end{array}$ & & $\begin{array}{l}88 \\
(64.2)\end{array}$ & & & & $\begin{array}{l}88 \\
(74.6)\end{array}$ & & $\begin{array}{l}109 \\
(87.9)\end{array}$ & & & & & Controls $<\mathbf{0 . 0 0 1}$ \\
\hline
\end{tabular}

Abbreviations: $\mathrm{df}=$ degree of freedom; $\mathrm{N}=$ number; $\mathrm{sd}=$ standard deviation 


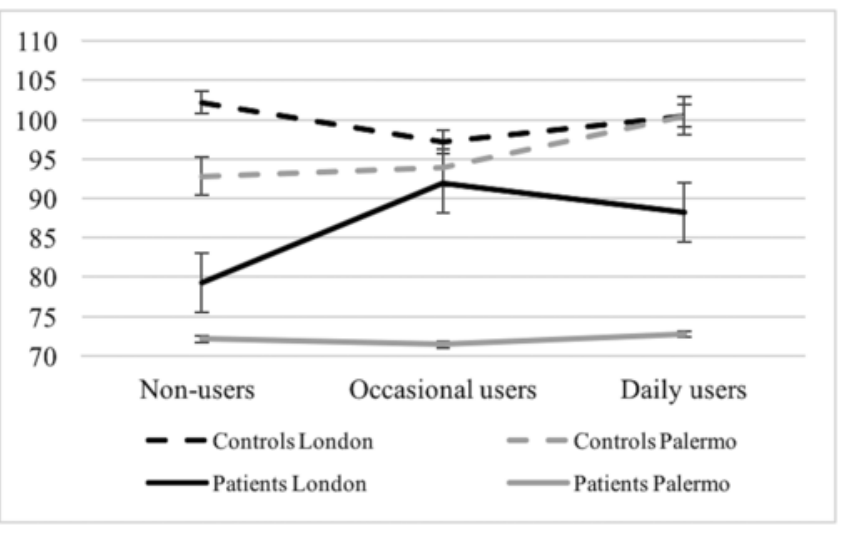

Fig. 1. IQ in patients and controls from London and Palermo, by frequency of cannabis use. Legend: the graph reveals the interaction effect between group, city, and frequency of cannabis use, adjusted by education, ethnicity and living status.

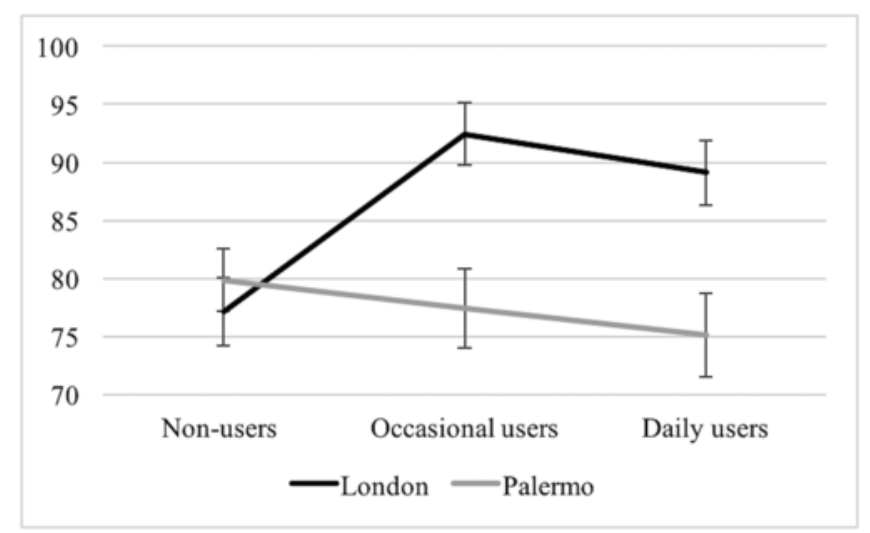

Fig. 2. Predicted IQ in patients from London and Palermo, by frequency of cannabis use. Legend: the graph reveals the interaction effect between city and frequency of cannabis use, adjusted by education, living status, negative symptoms and days of antipsychotic treatment at the moment of the IQ assessment.

(Aleman et al., 2003; McGrath et al., 2004; Morgan et al., 2008; Sideli et al., 2016a).

People in the two cities had different living status, with patients and controls from Palermo more likely to live with their parents, compared with London residents, maybe due to economic disadvantage (Eurostat. Statistical books, 2014) and to cultural expectations. Interestingly, this trend was more significant in patients than in controls within each city. Living alone has already been reported as a risk factor for psychosis in previous UK studies (Morgan et al., 2008; Stilo et al., 2013), while in Palermo we observed that living with their parents was more common among patients (68.1\%) (see also Sideli et al., 2016a). This latter could be a consequence of the higher economic vulnerability of these subjects, and the relative lack of financial support from the state but this could represent, in turn, a protective factor for psychosis (González-Pinto et al., 2011). Palermo had a lower proportion of patients $(3.4 \%)$ and controls $(10.3 \%)$ with academic education, compared to London patients (19.8\%) and controls (40.1\%); however, they had the same probability to be employed. The Palermo sample comprised white Caucasians and the 12 people from other ethnicities were all first-generation migrants, maybe because Sicily does not represent a desirable destination for migrant people who are looking for a job, given its high rate of unemployment (19.2\% in 2012) (Istituto Nazionale di Statistica, 2012).

\subsection{Differences in cannabis use}

As expected, lifetime cannabis use in London was similar in patients and controls, but patients were more likely to have used cannabis on a daily basis, compared to controls (Di Forti et al., 2009) and we found the same in Palermo. Another similarity between the two cities was age at first cannabis use, which occurred at a median age of 16 years in both the patient groups; an early age of cannabis use has been related to increased risk of schizophrenia (Arseneault et al., 2002) but it may be consequent to consumption of a bigger quantity of cannabis intake during the lifetime (Di Forti et al., 2009).

As hypothesised, we found a greater amount of cannabis-use in the London sample, compared to Palermo, especially in the patients' group. Patients from London were more likely than patients from Palermo to have used cannabis in their lifetime, on a daily basis, and to have chosen high potency cannabis $(57.4 \%$ of patients, $46.5 \%$ of controls) (Di Forti et al., 2015, 2014) Di Forti et al., 2019. In Palermo, only four patients and eight controls had ever tried high potency cannabis because the local drug market did not offer this type of cannabis at the time of the study. The mean THC concentration of marijuana in Italy at that time was around 4\% (Trends and developments 2013: Legal notice, 2013), much lower than the $14-16 \%$ reported for high potency cannabis commonly used in the UK (Hardwick, 2008). This could also help to explain the higher proportion of current smokers in patients and controls from London, given that high THC levels are associated with higher rates of dependence (Maldonado et al., 2011).

\subsection{Why do patients from Palermo have a lower IQ than patients from London?}

Our first analysis excluded the possibility that the difference between patients from the two cities reflected differences between the respective controls. Controls from Palermo appeared similar to their respective counterparts from London; once the IQ scores were controlled for several variables, they both had an IQ within the normal range (ranging 90-110). On the other hand, all patients from Palermo performed poorly on the IQ tests, irrespectively to cannabis use, while patients from London with cannabis use were more similar to their respective controls and controls from Palermo. London cannabis non-using patients were closer to patients from Palermo, regarding IQ.

Because of Italian laws regarding in-patient care, Palermo patients were tested within their first four weeks of hospitalisation, while patients from London were tested when they were less symptomatic (Ferraro et al., 2013). We, therefore, controlled for days of treatment with antipsychotics at the time of WAIS administration and differences in symptomatology in patients between the two cities, as it is known that negative symptoms can influence cognition (Bora et al., 2009) and treatment could improve cognitive performance, through improving positive symptoms (Trampush et al., 2015). We then obtained an interaction between cannabis use and city, where patients from London who never smoked cannabis showed very similar IQ scores to patients overall from Palermo, suggesting a stronger and more primitive influence of negative symptoms and education in this difference, than a role of the tratment. To allow the comparison of the different antipsychotic medications and dosages, we computed the dose-equivalent to $100 \mathrm{mg}$ of oral chlorpromazine (Gardner et al., 2010) (exploratory analysis). No differences emerged between London and Palermo $(\mathrm{t}(164)=1.3, \mathrm{p}=0.179)$ and no correlation was 
found between this measure and IQ $(R=-0.08, p=0.264)$; thus we decided to do not further correct the model for this variable.

\subsection{Are patients from Palermo a different population compared to patients from London?}

Incidence rates of psychosis in Palermo are much lower than those reported in London (Jongsma et al., 2018). Higher urbanisation density (4187 inhabitants $/ \mathrm{km}^{2}$ in Palermo and 5517 in London) and higher rates of migration in London, compared to Palermo, may have contributed to the higher incidence there (Mulè et al., 2017a).

Additionally, patients from London are more likely to live alone, while in Palermo they mostly live with their families; this could represent a difference in social cohesion between the two cities, more significant than the small difference in urbanisation density and a protective factor in itself.

Patients from Palermo were more likely to be diagnosed as "non-affective psychosis", thus their lower IQ could be related to these factors, as is known that people with non-affective psychosis perform poorly than those with affective psychosis (Krabbendam et al., 2005; Kravariti et al., 2009). Their lower intelligence (73.4) may also have been related to their lower levels of education (Ang et al., 2010), and by higher symptomatology at the time of the assessment. This finding is different from Italian studies on clinically stabilised patients, which found an average IQ of 84; however these studies excluded patients with an IQ lower than 70 and substance dependence or abuse (Bambini et al., 2016; Bechi et al., 2017), so their sample is not directly comparable to ours. Given that our principal interest was IQ, we did not exclude people with an IQ lower than 70 (traditionally considered in the intellectually disabled range). However, we corrected the analysis for education and symptoms, and this reduced the difference between the groups of patients without any cannabis use.

As our analysis considered all the risk-factors mentioned above as confounders, this allows us to speculate about the role of the greater prevalence of cannabis use, in London compared to Palermo; thus, this could have contributed in precipitating psychosis in a sub-group of patients with less neurodevelopmental impairment than in Palermo. This could also partially influence a higher incidence of psychosis in London, compared to Palermo (Jongsma et al., 2018), in line with findings on a previous study on the GAP sample in which the attributable population fraction of first-episode psychosis for skunk daily use in London was calculated at 24\% (Di Forti et al., 2015).

\subsection{Premorbid $I Q$}

Palermo patients had a normal premorbid IQ overall, in line with meta-analyses (Khandaker et al., 2011; Woodberry et al., 2008) and other studies that used TIB as a measure of premorbid IQ in schizophrenia (Piovan et al., 2016; Tosato et al., 2013). Premorbid IQ in patients in Palermo was higher in cannabis users than in non-users, in line with our previous study (Ferraro et al., 2013) but differently from a previous Italian study (Tosato et al., 2013), where no differences were detected, probably because these authors considered only cannabis use in the last 12 months (i.e. current use). However, differently from our previous study, we found a similar relationship in controls; this could be because patients from London include a higher percentage of less-vulnerable people exposed to a dangerous pattern of cannabis use, which generates a higher difference in IQ scores between cannabis and non-cannabis users in patients compared to controls, that we did not find in Palermo.

\subsection{Limitations}

The opportunity to test subjects from two different socio-cultural backgrounds is precious in exploring the role of environmental risk factors in psychosis, but this does involve more difficulties.

One issue could be differences in quality of education between the UK and Southern Italy. The Programme for International Student Assessment 2012 (PISA) shows that even if there is a difference in education achievement (lower in Italy), that we considered in our analysis, the two countries have slightly different scores for quality of reading, mathematics and science performance in 15-years-old students between 2003 and 2012. UK is in the country average (OECD) and a little below for mathematics (score 498.9), while Italy is a little below in all the three indicators (score 486.6) (PISA 2012, 2014). Interestingly, the two samples have comparable employment rates.

It is possible that we failed to detect a percentage of people affected by psychosis who did not look for psychiatric care, or who looked for a private psychiatrist because of the stigma (Mulè et al., 2017a; Sideli et al., 2016b); these could be the more economically advantaged people, with a higher IQ. However, stigma in psychosis is a generalised problem (Clement et al., 2015) and, at the moment, we do not have evidence that stigma in Italy is higher than in the UK. Other drug use and tobacco use could be confounders of our results. Tobacco use was scored as lifetime only. Thus we did not have the opportunity to use this variable in the analysis. As expected, use of other illicit drugs was higher in the UK compared to Italy at the time of the study (Trends and developments 2013: Legal notice, 2013). However, when we removed from the sample 11 subjects ( 7 patients and 4 controls) from London and 6 subjects (5 patients and 1 control) from Palermo who used stimulants (Chen et al., 2003) more than once a week for at least one year in their lifetime, our results did not change.

Information on frequency and type of cannabis use are not validated via biological measures, such as urine, blood or hair samples. However, these latter strategies do not allow reliable checking out for use over preceding years. Moreover, studies with both laboratory analyses and self-reported data confirmed that cannabis users reliably record the type of cannabis used (Freeman et al., 2014; Taylor et al., 2017). Previous results from the GAP study suggest that the accuracy of our self-reported data on cannabis use is high (Di Forti et al., 2009).

\section{Conclusions}

We found a greater cannabis use in the London sample, compared to Palermo. We also identified a higher IQ in patients from London, compared to patients from Palermo, but this difference was not identified between controls from the two cities.

Additionally, patients from London who had never smoked cannabis had similar IQ to patients from Palermo; thus the higher IQ scores of the London group overall were due to the subgroup of cannabis-using patients.

We can speculate that a greater amount of cannabis-use may have contributed by precipitating psychosis in patients with a higher IQ in London but less so in patients from Palermo.

\section{Contributor's statement}

Laura Ferraro: conception and design of the work, data collection, analysis and interpretation, draft of the article; Murray M. Robin and 
Daniele La Barbera: critical revision of the article, final approval of the version to be published; Marta Di Forti and Caterina La Cascia: data collection, critical revision of the article, final approval of the version to be published; Diego Quattrone, Giada Tripoli and Lucia Sideli: data collection, critical revision of the article.

\section{Role of the founding sources}

GAP study (London) was supported by the UK National Institute of Health Research Biomedical Research Centre grant (NIHR-BRC, SLAM). S-GAP study (Palermo) did not receive any specific grant from funding agencies in the public, commercial, or not-for-profit sectors. The funder had no role in study design, data collection and analysis, decision to publish, or preparation of the manuscript.

\section{Declaration of Competing Interest}

No conflict of interests to declare.

\section{Acknowledgments}

Special acknowledgment to all the patients and their families and controls who took part to the study. Thank you to the GAP study team at the Institute of Psychiatry, King's College London and to the research team of S-GAP at the Section of Psychiatry in Palermo (Dr Fabio Seminerio and all the psychiatrists in training) and to the colleagues working at the mental health-services of the Department of Mental Health of Palermo (ASP 6) and at Private Psychiatric Hospitals of Palermo.

\section{References}

Aleman, A., Kahn, R.S., Selten, J.-P., 2003. Sex differences in the risk of schizophrenia: evidence from meta-analysis. Arch. Gen. Psychiatry 60, 565-571.

Ang, S., Rodgers, J.L., Wänström, L., 2010. The Flynn effect within subgroups in the U.S.: gender, race, income, education, and urbanization differences in the NLSY-children data. Intelligence 38, 367-384. https://doi.org/10.1016/j.intell 2010.05.004.

Arseneault, L., Cannon, M., Poulton, R., Murray, R., Caspi, A., Moffitt, T.E., 2002. Cannabis use in adolescence and risk for adult psychosis: longitudinal prospective study. BMJ 325, 1212-1213.

Bambini, V., Arcara, G., Bechi, M., Buonocore, M., Cavallaro, R., Bosia, M., 2016. The communicative impairment as a core feature of schizophrenia: frequency of pragmatic deficit, cognitive substrates, and relation with quality of life. Compr. Psychiatry 71, 106-120. https://doi.org/10.1016/j.comppsych.2016.08.012.

Bebbington, P., Nayani, T., 1995. The psychosis screening questionnaire. Int. J. Methods Psychiatr. Res. 5, 11-19.

Bechi, M., Bosia, M., Spangaro, M., Buonocore, M., Cavedoni, S., Agostoni, G., Bianchi, L., Cocchi, F., Guglielmino, C., Smeraldi, E., Cavallaro, R., 2017. Exploring functioning in schizophrenia: predictors of functional capacity and real-world behaviour. Psychiatry Res. 251, 118-124. https://doi.org/10.1016/j. psychres.2017.02.019.

Bora, E., Murray, R.M., 2014. Meta-analysis of cognitive deficits in ultra-high risk to psychosis and first-episode psychosis: do the cognitive deficits progress over, or after, the onset of psychosis?. Schizophr. Bull. 40, 744-755.

Bora, E., Yücel, M., Pantelis, C., 2009. Cognitive functioning in schizophrenia, schizoaffective disorder and affective psychoses: meta-analytic study. Br. J. Psychiatry $195,475-482$.

Chen, C.K., Lin, S.K., Sham, P.C., Ball, D., Loh, E.W., Hsiao, C.C., Chiang, Y.L., Ree, S.C., Lee, C.H., Murray, R.M., 2003. Pre-morbid characteristics and co-morbidity of methamphetamine users with and without psychosis. Psychol. Med. 33, 1407-1414.

Clement, S., Schauman, O., Graham, T., Maggioni, F., Evans-Lacko, S., Bezborodovs N., Morgan, C., Rüsch, N., Brown, J.S.L., Thornicroft, G., 2015. What is the impact of mental health-related stigma on help-seeking? A systematic review of quantitative and qualitative studies. Psychol. Med. 45, 11-27. https://doi.org/10. 1017/S0033291714000129.

Di Forti, M., Morgan, C., Dazzan, P., Pariante, C., Mondelli, V., Marques, T.R., Handley, R., Luzi, S., Russo, M., Paparelli, A., Butt, A., Stilo, S.A., Wiffen, B., Powell, J., Murray, R.M., 2009. High-potency cannabis and the risk of psychosis. Br. J. Psychiatry 195, 488-491.
Di Forti, M., Sallis, H., Allegri, F., Trotta, A., Ferraro, L., Stilo, S.A., Marconi, A., La Cascia, C., Marques, T.R., Pariante, C., Dazzan, P., Mondelli, V., Paparelli, A., Kolliakou, A., Prata, D., Gaughran, F., David, A.S., Morgan, C., Stahl, D., Khondoker, M., MacCabe, J.H., Murray, R.M., 2014. Daily use, especially of high-potency cannabis, drives the earlier onset of psychosis in cannabis users. Schizophr. Bull. 40, 1509-1517. https://doi.org/10.1093/schbul/sbt181.

Di Forti, M., Marconi, A., Carra, E., Fraietta, S., Trotta, A., Bonomo, M., Bianconi, F., Gardner-Sood, P., O'Connor, J., Russo, M., Stilo, S.A., Marques, T.R., Mondelli, V., Dazzan, P., Pariante, C., David, A.S., Gaughran, F., Atakan, Z., Iyegbe, C., Powell, J., Morgan, C., Lynskey, M., Murray, R.M., 2015. Proportion of patients in South London with first-episode psychosis attributable to use of high potency cannabis: a case-control study. Lancet Psychiatry 2, 233-238. https://doi.org/10. 1016/S2215-0366(14)00117-5.

European Monitoring Centre for Drugs and Drug Addiction and Europol, 2016. EU Drug Markets Report: In-Depth Analysis, EMCDDA-Eur. ed, Publications Office of the European Union. Publications Office of the European Union, Luxembourg.

Eurostat. Statistical books, 2014. Summary for policymakers. In: Intergovernmental Panel on Climate Change (Ed.), Climate Change 2013 - the Physical Science Basis. Cambridge University Press, Cambridge, pp. 1-30. https://doi.org/10.1017/ CBO9781107415324.004.

Ferraro, L., Russo, M., O'Connor, J., Wiffen, B.D.R., Falcone, M.A., Sideli, L., Gardner-Sood, P., Stilo, S., Trotta, A., Dazzan, P., Mondelli, V., Taylor, H., Friedman, B., Sallis, H., La Cascia, C., La Barbera, D., David, A.S., Reichenberg, A., Murray, R.M., Di Forti, M., 2013. Cannabis users have higher premorbid IQ than other patients with first onset psychosis. Schizophr. Res. 150, 129-135.

Ferraro, L., Capuccio, V., Mulè, A., La Cascia, C., Sideli, L., Tripoli, G., Seminerio, F., Sartorio, C., La Barbera, D., Murray, R., Di Forti, M., 2016. Premorbid social adjustment is better in cannabis-using than non-using psychotic patients across Europe. Eur. Psychiatry 33, S102. https://doi.org/10.1016/j.eurpsy.2016.01.081.

Freeman, T.P., Morgan, C.J.A., Hindocha, C., Schafer, G., Das, R.K., Curran, H.V., 2014. Just say "know": how do cannabinoid concentrations influence users' estimates of cannabis potency and the amount they roll in joints?. Addiction 109, 1686-1694. https://doi.org/10.1111/add.12634.

Gardner, D.M., Murphy, A.L., O'Donnell, H., Centorrino, F., Baldessarini, R.J., 2010 International consensus study of antipsychotic dosing. Am. J. Psychiatry 167 686-693. https://doi.org/10.1176/appi.ajp.2009.09060802.

González-Pinto, A., de Azúa, S.R., Ibáñez, B., Otero-Cuesta, S., Castro-Fornieles, J., Graell-Berna, M., Ugarte, A., Parellada, M., Moreno, D., Soutullo, C., Baeza, I., Arango, C., 2011. Can positive family factors be protective against the development of psychosis?. Psychiatry Res. 186, 28-33. https://doi.org/10.1016/j. psychres.2010.05.015.

Hardwick, S., 2008. Home Office Cannabis Potency Study 2008. Home Off.

Harvey, P.D., Bowie, C.R., 2003. Cognitive deficits in schizophrenia: early course and treatment. Clin. Neurosci. Res. 3, 17-22.

Heinrichs, R.W., Zakzanis, K.K., 1998. Neurocognitive deficit in schizophrenia: a quantitative review of the evidence. Neuropsychology 12, 426-445.

Howes, O.D., Murray, R.M., 2014. Schizophrenia: an integrated sociodevelopmental-cognitive model. Lancet (London, England) 383, 1677-1687.

Istituto Nazionale di Statistica, 2012. ISTAT [WWW Document]. URL http://www. istat.it/it/istituto-nazionale-di-statistica, Accessed 30 June 2017.

Jongsma, H.E., Gayer-Anderson, C., Lasalvia, A., Quattrone, D., Mulè, A., Szöke, A., Selten, J.-P., Turner, C., Arango, C., Tarricone, I., Berardi, D., Tortelli, A., Llorca, P.-M., de Haan, L., Bobes, J., Bernardo, M., Sanjuán, J., Santos, J.L., Arrojo, M., Del-Ben, C.M., Menezes, P.R., Murray, R.M., Rutten, B.P., Jones, P.B., van Os, J., Morgan, C., Kirkbride, J.B., 2018. Treated incidence of psychotic disorders in the multinational EU-GEI study. JAMA Psychiat. 75, 36. https://doi.org/10.1001/ jamapsychiatry.2017.3554.

Kay, S.R., Opler, L.A., Lindenmayer, J.P., 1989. The positive and negative syndrome scale (PANSS): rationale and standardisation. Br. J. Psychiatry Suppl. 59-67.

Khandaker, G.M., Barnett, J.H., White, I.R., Jones, P.B., 2011. A quantitative meta-analysis of population-based studies of premorbid intelligence and schizophrenia. Schizophr. Res. 132, 220-227.

Krabbendam, L., Arts, B., van Os, J., Aleman, A., 2005. Cognitive functioning in patients with schizophrenia and bipolar disorder: a quantitative review. Schizophr. Res. 80, 137-149.

Kravariti, E., Morgan, K., Fearon, P., Zanelli, J.W., Lappin, J.M., Dazzan, P., Morgan, C., Doody, G.A., Harrison, G., Jones, P.B., Murray, R.M., Reichenberg, A., 2009. Neuropsychological functioning in first-episode schizophrenia. Br. J. Psychiatry $195,336-345$.

Lasalvia, A., Bonetto, C., Tosato, S., Zanatta, G., Cristofalo, D., Salazzari, D., Lazzarotto, L., Bertani, M., Bissoli, S., De Santi, K., Cremonese, C., De Rossi, M., Gardellin, F., Ramon, L., Zucchetto, M., Amaddeo, F., Tansella, M., Ruggeri, M., PICOS-Veneto Group, 2014. First-contact incidence of psychosis in North-Eastern Italy: influence of age, gender, immigration and socioeconomic deprivation. Br. J. Psychiatry 205, 127-134. https://doi.org/10.1192/bjp.bp.113.134445.

Maldonado, R., Berrendero, F., Ozaita, A., Robledo, P., 2011. Neurochemical basis of cannabis addiction. Neuroscience $181,1-17$. https://doi.org/10.1016/j. neuroscience. 2011.02.035. 
Marconi, A., Di Forti, M., Lewis, C.M., Murray, R.M., Vassos, E., 2016. Meta-analysis of the association between the level of Cannabis use and risk of psychosis. Schizophr. Bull. 42, 1262-1269. https://doi.org/10.1093/schbul/sbw003.

McGrath, J., Saha, S., Welham, J., El Saadi, O., MacCauley, C., Chant, D., 2004. A systematic review of the incidence of schizophrenia: the distribution of rates and the influence of sex, urbanicity, migrant status and methodology. BMC Med. 2, 13.

Mesholam-Gately, R.I., Giuliano, A.J., Goff, K.P., Faraone, S.V., Seidman, L.J., 2009. Neurocognition in first-episode schizophrenia: a meta-analytic review. Neuropsychology 23, 315-336.

Morgan, C., Kirkbride, J., Hutchinson, G., Craig, T., Morgan, K., Dazzan, P., Boydell, J., Doody, G.A., Jones, P.B., Murray, R.M., Leff, J., Fearon, P., 2008. Cumulative social disadvantage, ethnicity and first-episode psychosis: a case-control study. Psychol. Med. 38, 1701-1715.

Mulè, A., Sideli, L., Capuccio, V., Fearon, P., Ferraro, L., Kirkbride, J.B., La Cascia, C., Sartorio, C., Seminerio, F., Tripoli, G., Di Forti, M., La Barbera, D., Murray, R.M., 2017. Low incidence of psychosis in Italy: confirmation from the first epidemiological study in Sicily. Soc. Psychiatry Psychiatr. Epidemiol. 52, 155-162. https://doi.org/10.1007/s00127-016-1322-4.

Mulè, A., Sideli, L., Colli, G., Ferraro, L., Cascia, C. La, Sartorio, C., Seminerio, F., Tripoli, G., Forti, M. Di, Barbera, D. La, Murray, R., 2017. Cannabis consumption and the risk of psychosis. Evidence-based Psychiatr. Care 3, 25-31.

Murray, R.M., Quigley, H., Quattrone, D., Englund, A., Di Forti, M., 2016. Traditional marijuana, high-potency cannabis and synthetic cannabinoids: increasing risk for psychosis. World Psychiatry 15, 195-204. https://doi.org/10.1002/wps.20341.

Nelson, H.E., Willison, J., 1991. National Adult Reading Test (NART), Test Manual, 2nd ed. NFER Nelson, Windsor.

Piovan, C., Gava, L., Campeol, M., 2016. Theory of mind and social functioning in schizophrenia: correlation with figurative language abnormalities, clinical symptoms and general intelligence. Riv. Psichiatr. 51, 20-29. https://doi.org/10.1708/ 2168.23448 .

PISA 2012, 2014. Student performance in mathematics, reading and science. In: PISA 2012 Results What Students Know Can Do. vol.1, PISA, pp. 1-564. https://doi. org/10.1787/9789264208780-en.

Potter, D.J., Clark, P., Brown, M.B., 2008. Potency of $\Delta 9-$ THC and other cannabinoids in cannabis in England in 2005: implications for psychoactivity and pharmacology. J. Forensic Sci. 53, 90-94.

Potvin, S., Joyal, C.C., Pelletier, J., Stip, E., 2008. Contradictory Cognitive Capacities among Substance-Abusing Patients with Schizophrenia: A Meta-Analysis. vol. 100, 242-251.

Ringen, P.A., Vaskinn, A., Sundet, K., Engh, J.A., Jónsdóttir, H., Simonsen, C., Friis, S., Opjordsmoen, S., Melle, I., Andreassen, O.A., 2010. Opposite relationships between cannabis use and neurocognitive functioning in bipolar disorder and schizophrenia. Psychol. Med. 40, 1337-1347. https://doi.org/10.1017/ S0033291709991620.

Sartori, G., Colombo, L., Vallar, G., Rusconi, M.L., Pinarello, A., 1997. TIB. Test di intelligenza breve per la valutazione del quoziente intellettivo attuale e pre-morboso. La Prof. di Psicol. 1, 2-24.

Sideli, L., Mulè, A., La Cascia, C., Sartorio, C., Ferraro, L., Tripoli, G., Seminerio, F., Marinaro, A.M., La Barbera, D., 2016. Cumulative social disadvantage and psychosis:Findings from a southern Italy case-control study. In: Early Intervention in Psychiatry, Wiley, pp. 149-A107.

Sideli, L., Seminerio, F., Barone, M.V., Mulè, A., Cascia, C. La, Sartorio, C., D'Agostino, R., Ferraro, L., Tripoli, G., Francomano, A., Inguglia, M., Vassallo,
G., Majorana, C., Barbera, D. La, 2016. Perceived stigma in patients affected by psychosis: is there an impact on relapse?. Eur. Psychiatry 33, S257. https://doi.org/ 10.1016/J.EURPSY.2016.01.527.

Stilo, S.A., Di Forti, M., Mondelli, V., Falcone, A.M., Russo, M., O'Connor, J., Palmer, E., Paparelli, A., Kolliakou, A., Sirianni, M., Taylor, H., Handley, R., Dazzan, P., Pariante, C., Marques, T.R., Zoccali, R., David, A., Murray, R.M., Morgan, C., 2013. Social disadvantage: cause or consequence of impending psychosis?. Schizophr. Bull. 39, 1288-1295.

Tarricone, I., Mimmi, S., Paparelli, A., Rossi, E., Mori, E., Panigada, S., Carchia, G., Bandieri, V., Michetti, R., Minenna, G., Boydell, J., Morgan, C., Berardi, D., 2012. First-episode psychosis at the West Bologna Community Mental Health Centre: results of an 8-year prospective study. Psychol. Med. 42, 2255-2264. https://doi.org/10.1017/S0033291712000335.

Taylor, M., Sullivan, J., Ring, S.M., Macleod, J., Hickman, M., 2017. Assessment of rates of recanting and hair testing as a biological measure of drug use in a general population sample of young people. Addiction 112, 477-485. https://doi.org/10. 1111/add.13645.

Tosato, S., Lasalvia, A., Bonetto, C., Mazzoncini, R., Cristofalo, D., De Santi, K., Bertani, M., Bissoli, S., Lazzarotto, L., Marrella, G., Lamonaca, D., Riolo, R., Gardellin, F., Urbani, A., Tansella, M., Ruggeri, M., 2013. The impact of cannabis use on age of onset and clinical characteristics in first-episode psychotic patients. Data from the psychosis incident cohort outcome study (PICOS). J. Psychiatr. Res. 47, 438-444.

Trampush, J.W., Lencz, T., DeRosse, P., John, M., Gallego, J.A., Petrides, G., Hassoun, Y., Zhang, J.-P., Addington, J., Kellner, C.H., Tohen, M., Burdick, K.E., Goldberg, T.E., Kane, J.M., Robinson, D.G., Malhotra, A.K., 2015. Relationship of cognition to clinical response in first-episode schizophrenia spectrum disorders. Schizophr. Bull. 41, 1237-1247. https://doi.org/10.1093/schbul/sbv120.

Trends and Developments 2013: Legal Notice, .

Velthorst, E., Levine, S.Z., Henquet, C., de Haan, L., van Os, J., Myin-Germeys, I., Reichenberg, A., 2013. To cut a short test even shorter: reliability and validity of a brief assessment of intellectual ability in schizophrenia — a control-case family study. Cogn. Neuropsychiatry 18, 574-593. https://doi.org/10.1080/13546805. 2012.731390.

Ventura, J., Thames, A.D., Wood, R.C., Guzik, L.H., Hellemann, G.S., 2010. Disorganization and reality distortion in schizophrenia: a meta-analysis of the relationship between positive symptoms and neurocognitive deficits. Schizophr. Res. 121, 1-14. https://doi.org/10.1016/j.schres.2010.05.033.

Wechsler, D., 1997. WAIS-III Administration and Scoring Manual. The Psychological Corporation, San Antonio, TX.

Woodberry, K.A., Giuliano, A.J., Seidman, L.J., 2008. Premorbid IQ in schizophrenia: a meta-analytic review. Am. J. Psychiatry 165, 579-587.

World Health Organization, 1992. The ICD-10 Classification of Mental and Behavioural Disorders: Clinical Descriptions and Diagnostic Guidelines, WorldHealthOrganization, World Health Organization, Geneva.

World Health Organization, 1992. Schedules for Clinical Assessment in Neuropsychiatry (SCAN), World Heal, Geneva.

Yücel, M., Bora, E., Lubman, D.I., Solowij, N., Brewer, W.J., Cotton, S.M., Conus, P., Takagi, M.J., Fornito, A., Wood, S.J., McGorry, P.D., Pantelis, C., 2012. The impact of cannabis use on cognitive functioning in patients with schizophrenia: a meta-analysis of existing findings and new data in a first-episode sample. Schizophr. Bull. 38, 316-330. 